\title{
WAVELET BASED SPECTRUM SENSING TECHNIQUES For COGNITIVE RADIO - A SuRVEY
}

\author{
Karthik Divakaran ${ }^{1}$, Naveen Manikandan $\mathrm{P}^{2}$ and Shri Hari R R ${ }^{3}$ \\ Department of Electronics and Communication Engineering, \\ Amrita Vishwa Vidyapeetham, Ettimadai, Coimbatore, \\ India \\ ${ }^{1}$ divakaran.karthik@gmail.com \\ ${ }^{2}$ naveenp@live.com \\ ${ }^{3}$ r.r.shrihariegmail.com
}

\begin{abstract}
Spectrum scarcity is one of the major issues faced in Wireless communication technology. Efficient spectrum utilization is of utmost importance to alleviate the problem of interference and reduced data rates. Cognitive Radios adapt themselves according to the available spectrum and thereby enhance transmission and reception of data, without affecting adjacent band users. The pre-requisite for such an objective is the precise calculation of spectrum boundaries. Many methods have been suggested and revised from time to time. The Wavelet Edge Detection is one of the most widely used Spectrum Sensing techniques. This technique observes the spatial distribution of spectral data at multiple resolutions. The aim of this paper is to familiarize the reader with the mathematics behind the application of wavelets for edge detection, which is made use of for spectrum sensing applications. The several variants of this scheme which was originally formulated by Mallat et al are discussed and the inherent flaws or complexities are pointed out. The importance of choosing a suitable wavelet system is explained. We then proceed further and present an adaptive algorithm which chooses a suitable wavelet system by analyzing the nature of the spectrum. The slope of the Power Spectral Density is used as an index to distinguish between sharp and blunt peaks. Sparse spectra with conspicuous peaks utilize Haar wavelet system whereas dense spectra with subtle peaks use Gaussian Wavelet System. Multi-scale sums are used since they produce more accurate results than multi-scale products. The simulations are carried out in the FM frequency band (88-108 $\mathrm{MHz}$ ) using MATLAB.
\end{abstract}

\section{KEYWORDS}

Cognitive Radio, Spectrum Sensing, Edge Detection, Continuous Wavelet Transform, Multi-Scale Product, Multi-Scale Sum.

\section{INTRODUCTION}

In Cognitive radio technology, Spectrum sensing is critical for efficient utilization of the given bandwidth. By precisely locating the spectrum boundaries one can effectively prevent the problem of interference between adjacent bands and increase the data rates in the channel. Of various attempts that have been made to accurately detect the spectral boundaries, Wavelet Edge detection is a method which is widely utilized; however the efficiency of this system is highly dependent upon the type of wavelet used. In this paper we propose an adaptive spectrum sensing technique which chooses the optimum wavelet function for the given spectrum after analysis of the nature of peaks in the power spectral density of the spectrum. The traditional techniques use a particular wavelet function for the problem of edge detection as explained in [1], followed by energy detection or periodicity detection [2], [3]. But a single wavelet function cannot be effectively used for the real time spectrum data whose PSD characteristics differ 
vastly. Hence an adaptive algorithm for analyzing the spectral characteristics and choosing the optimum wavelet function for better edge detecting capability is proposed in this paper. For the regions in the PSD which has sharp and pointed peaks, Haar wavelet function is utilized and for the regions in the PSD which shows gradual variations in the peaks, Gaussian wavelet function is utilized. By obtaining the continuous wavelet transform of the spectrum's PSD using the multi-scale versions of the chosen wavelet, edge detection is achieved by noting the local extrema of the modulus first derivative of the smoothed signal. This method of adapting to the slope characteristics of the PSD data has proven to yield better edge detection and thus to estimation of spectral boundaries with a greater resolution. By calculating the energy of the obtained sub-bands, precise information on spectral occupancy is ascertained. This paper proposes an adaptive wavelet based edge detection technique as a modification to the traditional Spectral Sensing techniques proposed in [4], [5].Simulated FM spectrum is used to test the performance of this approach.

The rest of the paper is organized as follows. Section 2 briefs the Traditional Spectrum Sensing methods that were in use prior to Wavelet based methods. In Section 3 the theory behind Wavelet Edge detection is explained in detail. This is followed by a review of currently used Spectrum Sensing techniques employing Wavelets. In Section 5 our new modified algorithm is presented. Section 6 contains the results of edge detection on different types of FM signals. Finally, Section 7 contains the summary of the paper.

\section{Traditional Spectrum Sensing Methods}

Spectrum sensing is essentially a case of energy detection, where the presence or absence of meaningful data at a particular frequency band is to be found out. Presence of data implies an increase in Energy from the noise floor, or the presence of some noise-like signal with higher order periodicity. Traditional approaches operate in narrowband and consist of a series of FIR filters which are tuned for the particular frequency range. While this approach is good enough for narrow bandwidths, it is found inefficient when the dynamic range of operation of Cognitive Radios increases. For ultra-Wideband Cognitive radio systems, this approach has very high complexity. However, to understand the features of Wavelet based algorithms, it is suggested that the reader has first-hand knowledge on the traditional methods, so that the discussion is directed only towards those aspects of Spectrum Sensing which are not achieved using these methods.

\subsection{Short-Time Fourier Transform (STFT)}

This method is also called as 'Windowed Fourier Transform'. Generally, Fourier Transform is a transformation from time domain to frequency domain, where the time averaged values for various frequency components are obtained. While this technique can be useful for analyzing the frequency components, we cannot determine their time of occurrence. i.e time localization of frequencies cannot be obtained. In short time fourier transform, the entire signal is divided into smaller segments (using a suitable window function) and the Fourier Transform is obtained for these intervals. The formula for STFT is given below:

$$
X(T, f)=\int_{t}[x(t) u(t-T)] e^{-j 2 \pi t+} d t
$$

While this method provides better results than Fourier analysis, it suffers because of low frequency resolution, high variance of estimated power spectrum and high side lobes/leakages.

\subsection{Periodogram}

The Periodogram was one of the most commonly used Spectrum Sensing techniques until better methods were developed to replace it. In this method, the infinite length sequence is truncated using a rectangular window function, and the FFT is obtained. Square of FFT gives an 
approximate Spectral Density plot. The major issue with this method is because of the abrupt truncation of the signals. As explained in [6],[7] this results in a Dirichlet Kernel in the frequency domain, described by the width of main lobe and side lobes. This in turn leads to spectral leakage at the discontinuities. Also, this method failed to provide time-frequency localization.

\subsection{Matched Filter Approach}

This is a pilot detection method. This is the fastest approach for spectrum sensing, but fails because of the fact that prior knowledge about the primary user's modulation type, pulse shaping and packet format is required. Timing and synchronization is essential to achieve coherence. But due to channel fading effects, there is a chance for time dispersion and Doppler shifts which ultimately affect synchronization. There is much literature available in [8] regarding the implementation of matched filters.

\subsection{Cyclo-stationary Feature Detection}

Any type of observable periodicity can be considered as first order periodicity. In the transmitted data, because of the modulation techniques or due to source coding, a certain periodicity is added to the signal which can be observed only through non-linear time invariant transformations of the time series[9]. This type of second order periodicity is called cyclostationarity. Generally the mean, autocorrelation and other statistical features show periodic behaviour. This can be exploited to determine the presence or absence of data in a frequency band. The advantage is that, this is the only method which provides accurate information about spectral occupancy in very low SNR bands. However the boundaries cannot be accurately determined. Hence this technique combined with boundary detection is often used for developing algorithms. A detailed treatment about computing the Cyclic Spectrum Density is available in [10].

\subsection{Multi Taper Spectrum Estimation}

The problems faced in the Periodogram approach are slightly alleviated in this method. The Spectral leakage and variance of the estimated power are reduced using multiple orthogonal filters. Consider the received signal $X(n)=[x(n) x(n-1) \quad x(n-2) \ldots \ldots . . x(n-M+1)]^{T}$ consisting of $M$ samples. An orthogonal basis is formed using these data points and the expansion coefficients are varied using a set of values which denote the way the spectrum tapers. A detailed formulation is presented in [11]. It can be inferred that MTSE is the average of several Periodograms with different windows. Thus each window shape reveals different aspects of the spectrum and the averaged value smoothens the discontinuous points thereby reducing spectral leakage. This method suffers because of excessive computations and the fact that this cannot completely alleviate the problem of Spectrum leakage while better methods can.

\subsection{Quadrature Mirror Filter Banks}

In this method, the entire wideband spectrum is split into M-bands. These are pre-defined bands where user traffic is monitored using $\mathrm{n}$ stages of Quadrature Mirror Filters tuned for the particular band, such that $M=2^{\mathrm{n}}$. Thus, a prior knowledge is required about the Primary user is required as is the case in Matched Filter Method. However, since a set of Filter banks are used, these same filters can be used for data reception after sensing spectral holes [12]. Thus they serve dual purpose. Further the tree structure helps reduce the computational complexity. The energy detection begins from the first stage where there are only 2 filters. Thresholds are fixed to determine whether to proceed to the next stage or not. If the signal energy is greater than threshold, then the process of analysing the sub-bands is omitted. The problem with this approach is that, spectrum whose boundary does not coincide with the assigned pass-band of a particular filter, will lead to unnecessary wastage of free spectra. Thus this technique is not 
capable of identifying spectral holes narrower than the pass-band of the last stage filter. Also, the channel fading effects greatly affect its performance.

These techniques and their inherent flaws propelled researchers to deduce a domain transformation technique where both spatial and spectral data could be analysed simultaneously. Wavelets emerged as a promising concept with great potential in resolving these aforementioned issues. They provided time frequency localization to a great extent. Also, their response to function discontinuities (singularity) allowed researchers to utilize them for many boundary detection applications. In the next section, the Mathematics behind Wavelet Edge Detection is discussed.

\section{WAVELET THEORY OF EDGE DETECTION}

Before proceeding to the analysis of Edge Detection using wavelets, it is beneficial to present a brief introduction to the development of the Wavelet theory. This is given in the next subsection followed by the Edge detection technique.

\subsection{Development of Wavelet Analysis}

The concept of wavelet analysis has been developed since the late 1980's. Wavelet analysis is a powerful tool for time-frequency analysis. Fourier analysis was believed to be a good tool for frequency analysis, but later, after close examination it was revealed that they can only provide global frequency information, which is independent of time. Hence, with Fourier analysis, it is impossible to describe the local properties of functions in terms of their spectral properties. This can be viewed as an expression of the Heisenberg uncertainty principle which implies that high time resolution and high frequency resolution cannot be obtained at the same time. This was one of the major drawbacks of Fourier transforms. Moreover, Fourier transforms cannot exactly depict the signals which have high transient response i.e. signals with abrupt changes. In many applied areas like digital signal processing, time-frequency analysis is critical. That is, we want to know the frequency properties of a function in a local time interval.

Overcoming the drawbacks of the Fourier analysis, wavelets provide an efficient way to perform time-frequency analysis. First, a mother wavelet is chosen i.e. a function subject to some conditions like mean-value 0. Instead of using pure harmonics as in Fourier analysis, wavelets use shifted and dilated versions of the mother wavelet. By using a two variable base: one for the amount of shift and the other for the amount of dilation, we can introduce enough redundancy to maintain the local properties of the original function. This makes wavelets desirable in places in which Fourier transforms formerly yielded poor results. This also provides desired quality of time-frequency resolution compared to other transforms.

The Wavelet transform is a way of decomposing a signal of interest into a set of basis waveforms, called wavelets, which thus provide a way to analyze the signal by examining the coefficients of wavelets. In most of the applications, the power of the transform comes from the fact that the basis functions of the transform are localized in time and frequency, and have different resolutions in these domains. A formal definition for the Continuous Wavelet Transform is given below.

The continuous wavelet transform (CWT) of a signal $x(t)$ is defined as the sum of all time of the signal multiplied by scaled, shifted versions of the wavelet $\phi(t)$. This is expressed as:

$$
W(a, b)=(1 / \sqrt{ } a) \int_{-\infty}^{\infty} x(t) \varphi((t-b) / a) d t
$$

Where,

a- Scaling factor

b- Shifting factor 
Original signal can be reconstructed using the inverse transform formula,

$$
x(t)=(1 / C \psi) \int_{-\infty}^{\infty} W(a, b) \varphi((t-b) / a) d a\left(d b /\left|a^{2}\right|\right)
$$

where,

$$
C \psi=\int_{-\infty}^{\infty}\left(|\psi(\omega)|^{2}\right) /|\omega| d \omega \text { and } \psi(\omega) \text { is the Fourier transform of } \phi(t) .
$$

The CWT is often used for analysis of continuously time varying properties of Signals. A discretised version of this transform (Discrete Wavelet Transform) is generally used for filtering, denoising and compression [13].

\subsection{Wavelet Edge Detection}

Points of singularities are important features for analysing properties of transient signals. For example, in image signals they are generally located at the boundaries of important image structures. In order to detect these edges several researchers have proposed multiscale edge detection techniques. Wavelet transform method gives deeper understanding of these edge detection algorithms. The scale in the multiscale edge detection defines the size of the locality where the signal variations are computed. Edge detection can be done in two ways one is using the canny edge detector which is equivalent to finding the local maxima of the wavelet transform modulus of the signal and the other method is to use Marr-Hildreth edge detector where the singularities are characterised as zero-crossings of the second derivative of the wavelet transforms. There may be various points in the given signal where the singularities are present. To detect these edges one has to compute the local properties of the signal singularities. These singularities corresponding to edges can be characterised by lipschitz exponents. Using the properties of wavelets these lipschitz exponents can be computed from the evolution across different scales of wavelet transform maxima modulus. A numerical procedure exists to measure these exponents. The nature of the smoothness of the edge can also be calculated by the degradation of wavelet transforms across different scales which give profound information about the intensity profiles of different types of edges in the signals.

In original multiscale edge detection [1], a smoothing function $\Theta(x)$ whose integral is 1 and converges to 0 at infinity is defined. An example for such $\Theta(x)$ is a Gaussian function. Let $\Phi_{1}(x)$ and $\Phi_{2}(\mathrm{x})$ be the first and second derivative of $\Theta(\mathrm{x})$. $\Phi_{1}(\mathrm{x})$ and $\Phi_{2}(\mathrm{x})$ can be defined as wavelets because their integral is 0 .

$$
\begin{aligned}
& \int \Phi 1(x)=0, \int \Phi 2(x)=0 \\
& \Phi 1(x)=d[\theta(x)] / d x \\
& \Phi 2(x)=d^{2}[\theta(x)] / d x^{2}
\end{aligned}
$$

A Scaling function $\varepsilon$ is defined such that

$$
\varepsilon_{s}(x)=(1 / s) \varepsilon(x / s)
$$

with a scaling factor $\mathrm{s}$.

A wavelet transform is obtained by convolving the given signal with the dilated wavelet scaled by $\mathrm{s}$. Wavelet transform of a signal $\mathrm{f}(\mathrm{x})$ is obtained as in

$$
\begin{aligned}
& W_{s}^{a} f(x)=f(x) * \Phi_{1, s}(x) \\
& W_{s}^{b} f(x)=f(x) * \Phi_{2, s}(x)
\end{aligned}
$$


Where $*$ is the convolution operator. It is derived that,

$$
\begin{aligned}
& W_{s}^{a} f(x)=f * s \frac{d[\theta s(x)]}{d x}=\frac{d[f * \theta s(x)]}{d x} \\
& W_{s}^{b} f(x)=f * s^{2} \frac{d^{2} \theta s(x)}{d x^{2}}=s^{2} \frac{d^{2} f * \theta s(x)}{d x^{2}}
\end{aligned}
$$

The wavelet transforms $\mathrm{W}_{\mathrm{s}}^{\mathrm{a}}$ and $\mathrm{W}_{\mathrm{s}}^{\mathrm{b}}$ are respectively the first and the second derivative of the signal $f(x)$ smoothed by $\Theta_{s}(x)$. The local extrema of $\mathrm{W}_{\mathrm{s}}^{\mathrm{a}}$ are the zero crossings of the second derivative $\mathrm{W}_{\mathrm{s}}^{\mathrm{b}}$ and also correspond to the points of inflection points of $\mathrm{f}(\mathrm{x})$ smoothed by $\Theta_{\mathrm{s}}(\mathrm{x})$ [i.e. $f^{*} \Theta_{\mathrm{s}}(\mathrm{x})$ ]. When the scale $\mathrm{s}$ used is large, convolution with wavelet function removes the small fluctuations and denotes only the sharp peaks or variations in the given signal. The inflection points of $f^{*} \Theta_{s}(x)$ can either be a maximum or minimum of $\left|W_{s}^{a} f(x)\right|$. The maxima of $\left|\mathrm{W}_{\mathrm{s}}^{\mathrm{a}} \mathrm{f}(\mathrm{x})\right|$ indicate sharp variations of $\mathrm{f}^{*} \Theta_{\mathrm{s}}(\mathrm{x})$ whereas a minima of $\left|\mathrm{W}_{\mathrm{s}}^{\mathrm{a}} \mathrm{f}(\mathrm{x})\right|$ only slow variations. With a second derivative operator it is difficult to recognize these points of inflection in terms of zero crossings. However, on a first order operator it is easy to detect sharp peaks by observing the local maxima of $\left|\mathrm{W}^{\mathrm{a}}{ }_{\mathrm{s}}\right|$. Hence $\mid \mathrm{W}_{\mathrm{s}}^{\mathrm{a}} \mathrm{l}$ on different scales is widely used for edge detection problems.

\subsection{Edge detection using dyadic wavelet transforms}

In most cases of spectrum sensing problems, usage of continuous parameter "s" is not favourable. And hence to impose fast numerical executions dyadic scaling is used [14]. In dyadic edge detection, the continuous dilation parameter " $\mathrm{s}$ " is redefined as: $\mathrm{s}=2^{\mathrm{j}},(j=1,2, \ldots)$ i.e. the scale varies only as a dyadic sequence and not continuously. A wavelet function $\Psi$ whose average is 0 according to the formal definition of wavelet is used. Hence a new wavelet representation using $\Psi$ with the dyadic scale is represented as

$\Psi_{2 j}(x)=\left(1 / 2^{j}\right) \Psi_{2 j}\left(x / 2^{j}\right)$

The wavelet transform using the dyadic scale is represented as

$W_{2 j} f(x)=f * \Psi_{2 j}(x)$

Therefore dyadic wavelet transforms are a sequence of functions which varies with dyadic scale of $2 \mathrm{j}$ and are represented by

$$
\boldsymbol{W} f=W_{2 j} * f(x)_{j \in Z}
$$

Where $\boldsymbol{W}$ is dyadic wavelet transform operator

From results obtained by former researchers, it is known that as we increase the scale $2^{\mathrm{j}}$ (number of dyadic wavelet transforms on the given signal) the information about the peak characteristics of the signal is compromised. To overcome this problem, integration of the multiscale transforms is done and the multiscale products [5] and multi-scale sums [2] are obtained.

Multi-scale product is given by:

$\mathrm{P}_{j} f(x)=\prod_{j=1}^{J} W_{s}^{a} f(x)$

Multi- scale sum is given by: 
$S_{j} f(x)=\sum_{j=1}^{J} W_{s}^{a} f(x)$

Where $\mathrm{J}$ is the upper limit for scale $\mathrm{j}$ in $\Psi_{2 \mathrm{j}}$.

\section{REVIEW OF WAVELET BASEd SPECTRUM SEnSING TECHNIQUES}

\subsection{Multi-scale Product}

This method involves finding discontinuities in PSD of the signal by taking the multiscale wavelet transforms and estimating the edges as explained in Section 3.3. This is done under the assumption that the discontinuities in the Power Spectral Density represent the spectral boundaries. The energy of each sub-band is calculated for spectrum occupancy. This method also assumes that there is always an empty sub-band and hence noise band can be determined. Taking multiscale product of the wavelet transform or its derivative at different scales enhances the multiscale peaks due to edges while suppressing the noise. To obtain better results for edge detection, using small values of scale 's' is advisable [15]. This happens only when the spectral characteristics of the channel changes abruptly. Therefore in the real time spectra where the peaks are non-ideal and not abrupt, usage of CWT is preferable than using the derivative of CWT for edge detection. Using a fixed set of scales for multiscale product may result in weak correlation with the dilated wavelet function. To avoid this, a new set of scale representation is introduced where the scales are taken in multiple groups. By taking multiple groups of scales it is possible to estimate each boundary by each group of scale.

\subsection{Multi-scale Sum}

Theoretical analyses indicate that different signals have distinct cross scales information at dissimilar scales [5]. Hence the wavelet transforms at different scales give important information about lipschitz exponents at the sharp variation points. Since the real spectrum consists of non-ideal characteristics due to various multipath transmissions and Doppler shifts their edge characteristics are not uniform. As the scale is increased abrupt changes in the spectra result in singularities in the wavelet transforms that are large and in regions of slow variation singularities in the wavelet transforms are smaller. Taking multiscale product in a certain range $(a, b)$ yields interesting results. In the lower range with scales $s=1,2,3$ the transform gives peaks for abruptly varying functions and in higher range with scales $s=3,4,5$ the peaks are conspicuous for gradual varying slopes. This is because, when processing DWT, the lower scale component corresponds to high frequency variation while the higher scale component corresponds to low frequency variation, therefore slow varying PSDs correspond to small peaks in multiscale product so they have a dominating effect to suppress the peaks when multiscale product is taken. This dominating effect produces pulse suppression which leads to inefficient edge detection. Hence due to this suppressing effect multiscale sums are preferred to multiscale products. Multiscale sum over large range gives the most accurate edge detection capabilities. But when the sum is taken over a large range singularity information may be compromised, therefore literature [5] concludes that multiscale sum and multiscale product must be used combination to obtain best edge detection.

\subsection{DWPT approach}

The Discrete Wavelet Packet Transform described below is a better edge detection method than Continuous Wavelet Transform (CWT) based methods given above. The wavelet packet transform is just like the wavelet transform except that it decomposes even the high frequency bands which are kept intact in the wavelet transform. Figure 4 illustrates the Wavelet packet decomposition procedure. Here $S$ denotes the signal while $A$ and $D$ denote the respective approximations (high frequency terms) and decompositions (low frequency terms). This is illustrated in the Figure shown below [16]. 


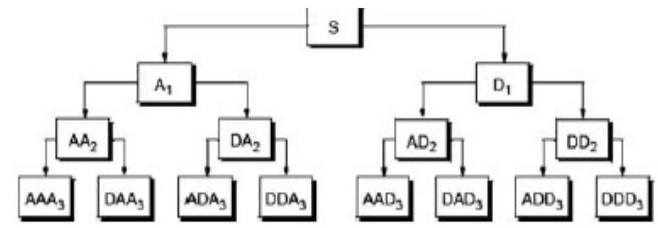

Figure 4. Wavelet packet decomposition tree of input signal [15].

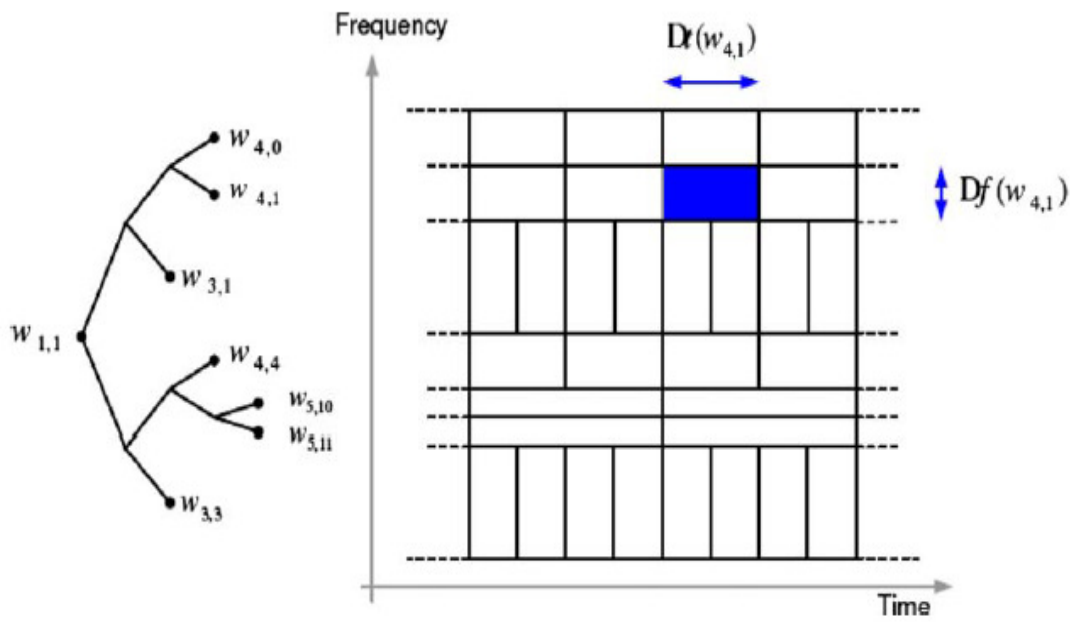

Figure 1. Wavelet packets; (Left): Semi-arbitrary tree pruning; (Right): Time-frequency plane division Here $w_{i, j}$ denotes the $j$ th wavelet packet coefficient at $i$ th iteration level and $D_{t}\left(w_{i, j}\right)$,

$D_{f}\left(w_{i, j}\right)$ denote the length of the time and frequency division, of the wavelet packet $w_{i, j}$.

Transceivers based on Wavelet Packet Transform have been implemented for adaptively activating or vacating sub-carriers in a way that utilizes unoccupied time-frequency gaps of the Primary user. In [17], Wavelet Packet Multi-Carrier Modulation Transceiver architecture is explained in detail, which makes use of DWPT and its inverse transform for dynamic bonding with unoccupied spectrum bands. The Wavelet Packet Transform of the received data is obtained and the spectrum is estimated from it. The wavelet packet coefficients are ordered using Gray codes because of the Low/High pass filtering tree structure. Based on preset threshold levels, the bands are classified as occupied or unoccupied. Transmitted Waveform Shaping is done in such a way that frequency bands in the vicinity of the Primary user are left alone and vacant bands are used. Thus, the entire operation is made invisible to the primary user.

Even though this method gives better results in AWGN channel, the performance deteriorates when subjected to fading channels. Also, this method is demonstrated for low ISI/ICI environments. The performance of this method in High Interference environments and in OFDM systems is not verified.

\subsection{Compressed wideband sensing approach}

In open spectrum access, cognitive radios must rapidly tune their transmitter parameters to utilize the available spectrum efficiently. This calls for fast and accurate spectrum sensing over a wide bandwidth, which challenges traditional spectral estimation methods operating at or above Nyquist rate. This technique capitalizes on the sparseness of the spectrum in open access method and develops compressed sensing techniques suitable for of spectrum hole identification where coarse sensing would suffice. This method mainly focuses on improving the speed of Spectrum Sensing by sub-Nyquist 
sampling followed by non-linear signal reconstruction. The method under discussion facilitates fast spectrum sensing at affordable complexity. A two step approach is used for spectral classification. Coarse spectrum sensing is done to detect non-overlapping spectrum bands and classify them into black, gray or white spaces, depending on whether the power spectral density (PSD) levels are high, medium or low. Based on the spectrum sharing mechanism adopted, the second stage of fine-scale spectral shape estimation is performed only when needed, and mostly confined within the available (narrowband) white spaces to alleviate the sampling requirements.

Random sub-Nyquist-rate samples are used to formulate an optimal signal reconstruction problem, which incorporates the wavelet-based edge detector to recover the locations of frequency bands. Because spectrum location estimation takes priority over fine-scale signal reconstruction, this method is robust to noise and can afford reduced sampling rates. A detailed explanation regarding sub-Nyquist sampling is provided in [18].After the reduction of the number of samples spectrum reconstruction is performed using a Basis Pursuit (BP) technique [19] and then the normal edge detection routine is followed for sensing the reconstructed spectrum.

As explained previously, this method is only applicable for sparse spectra and not for closely packed channels. Further, the random selection of samples to be discarded need not necessarily be distributed. If the selected points are closely spaced, there is a possibility for aliasing to occur, thereby deteriorating the spectral information.

Having discussed several approaches to spectrum sensing in CR, we can observe that any further research in this regard must be directed towards the implementation aspect of the algorithms. There is a wide gap between theoretical propositions and practical implementations. This gap can be narrowed by exploring the circuit level optimizations and custom-made hardware implements possible. Also, there is a need to develop algorithms which can adaptively sense dynamic spectrum under varied environments. With this in mind, we present a novel algorithm which provides accurate results for the edge detection problem by adapting according to the characteristics of the spectrum.

\section{Proposed Method}

Continuous wavelet transform can be considered as the correlation between shifted and scaled versions of the mother wavelet with the signal in hand. In other words, it measures the similarity between the mother wavelet and the signal, at different spatial resolutions. In the proposed method, we study the characteristics of the spectrum in order to choose as to which wavelet system shows resemblance to the general spatial features of the signal. A chosen set of wavelets is used as the basis for the sensing operation. The proposed algorithm is as follows:

1. The spectrum data, $S_{r}(f)$ is smoothened using a moving average filter in order to remove the disturbances. If there is a possibility for the presence of occupied bands with very low SNRs, this step can be neglected.

$S_{r s}(f)=\left(S_{r}(f-1)+S_{r}(f)+S_{r}(f+1)\right) / 3$

2. The smoothened spectrum, $S_{r s}(f)$ is taken in an array, $a$. Another set, $b$ is obtained by taking the difference of two points in the spectrum such that the minimum distance between the two frequencies is less than half the bandwidth of the smallest channel. This is to invalidate the randomness generated in the signal due to the presence of noise. For the remaining frequencies, suitable redundancies are added. 
3. The actual spectrum is compared with the difference set generated. If the peaks in actual spectrum lie between the peaks in the difference set then proceed to step 4 or else, repeat step 2 by increasing the frequency difference, in such a way that the condition mentioned in step 2 is met.

4. The absolute difference values in the difference set are compared with a fixed threshold, $\delta$.

Haar Wavelet System is chosen if

$S_{r s}^{\prime}(f) \geq \delta$

else, Gaussian wavelet system is used.

5. The Multi-scale sum is computed using dyadic scales for both the wavelet systems.

6. Individual frequency points are checked for the condition in (15). Accordingly, a composite data set consisting of peaks derived from both the Multi-scale sums is obtained.

7. Each local maxima in the composite data set denotes a spectrum boundary.

The advantage of using this method is that, even peaks which are weakly correlated to a particular Wavelet system can be detected by using another wavelet system. The use of dyadic scales has problems when there is excessive noise. This can be removed by using two different functions with compact support, so that the peaks affected by noise in one wavelet system can be observed in some other wavelet system. Also, the location of boundaries can be more accurately predicted since the best wavelet system will be used for sensing a particular boundary. The simulation results for the current methods as well as the proposed method are presented in the next section.

\section{SimUlation RESUltS AND DisCUSSIONS}

In this section, 3 different simulated spectra in the FM frequency range are chosen for study. The FM frequency range lies between 88 and $108 \mathrm{MHz}$. Real-time data can be obtained using a Spectrum Analyser. The block diagram of the setup is given in Figure 1. For our case, MATLAB 2008 was used for the implementation of the algorithm and the wavelet coefficients were obtained using the standard Wavelet toolbox provided with MATLAB.

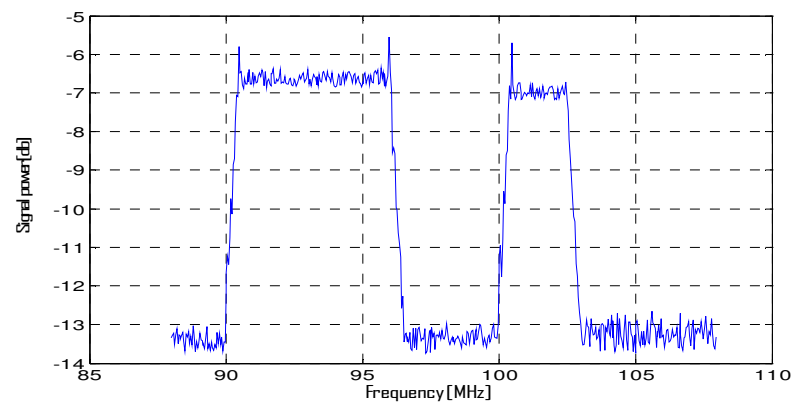

Figure 1. Simulated FM Spectrum with sharp boundaries

Figure 1. shows the simulated power spectral density plot for FM signals. The boundaries are very sharp and the peaks are sparse and large. The Haar wavelet system is especially suited for such spectra which are characterized by well defined edges [15]. In Fig.2 the coefficients at dyadic scales are shown. 


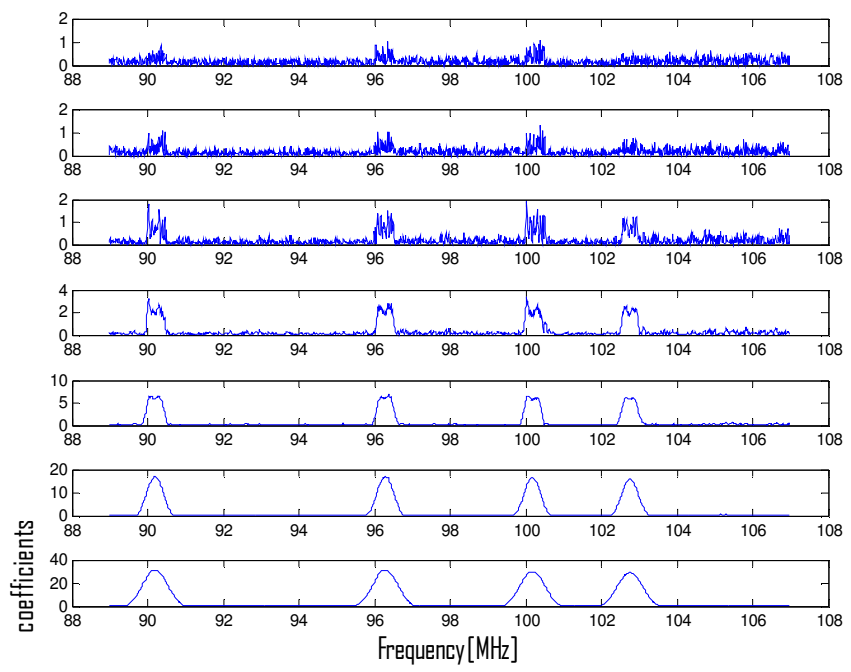

Figure 2. CWT coefficients at dyadic scales from top to bottom $(S=2,4,8,16,32,64,128)$

For this simulation Haar mother Wavelet, $\Phi_{\text {Haar }}$ was used.

It can be observed that the peaks are clearly visible in all the scales of dilation. The multi-scale product suggested in [15], and the multi-scale sum explained in [5] is plotted in Figure 3 below.
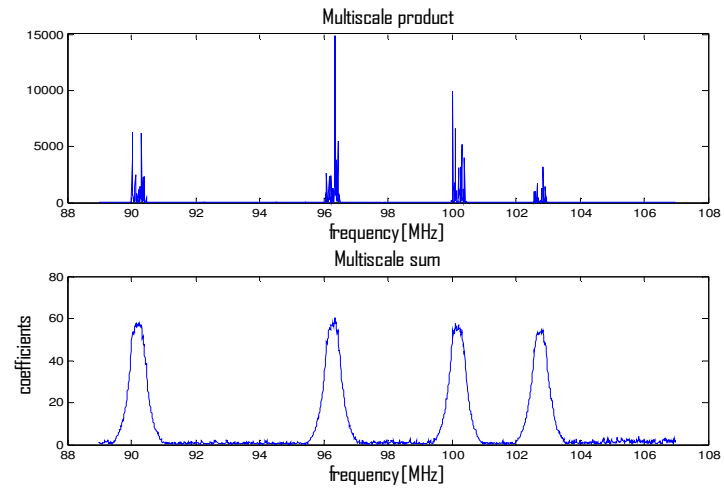

Figure 3. Multi-Scale Product(Top);Multi-Scale Sum(Bottom)

It can be observed that multi-scale sum gives more accurate results than multi-scale product. This is because, when we take product, the peaks in one scale are suppressed by near-zero values of coefficients in some other scale. Hence for majority of Spectra, the multi-scale sum works well. The observed spectral boundaries for the Sample spectrum-I using Haar Wavelet system were [90.22, 96.35, 100.1, 102.8] MHz respectively.

In the next Figure, another type of Spectrum is simulated. This figure depicts envelopes which are blunt or which cannot be separated through direct inspection. Haar wavelet system is inefficient for such cases. Hence Gaussian wavelet system is used. Figure 4 depicts the spectrum before passing through the Smoothing filter (moving average filter). 
International Journal of Computer Science \& Information Technology (IJCSIT), Vol 3, No 2, April 2011

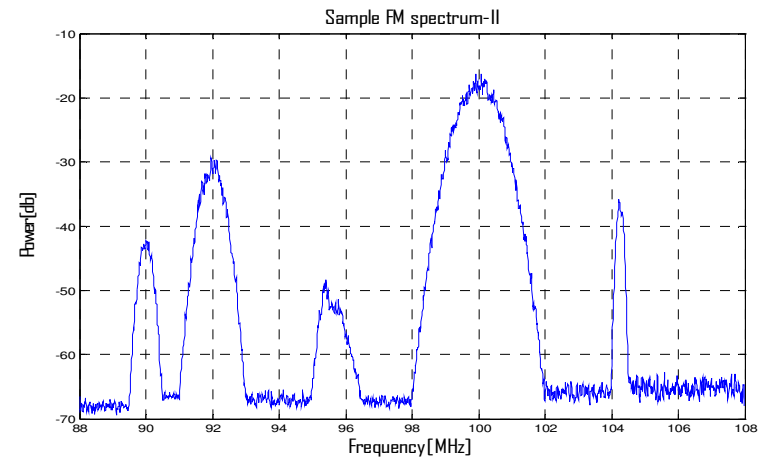

Figure 4.Simulated FM Spectrum with blunt envelopes

In Figure 5, the different scale coefficients are shown and in Figure 6, the Multi-scale Product and Sum are plotted. Even for this spectrum, Multi-scale sum provides better approximation for the spectral boundaries.

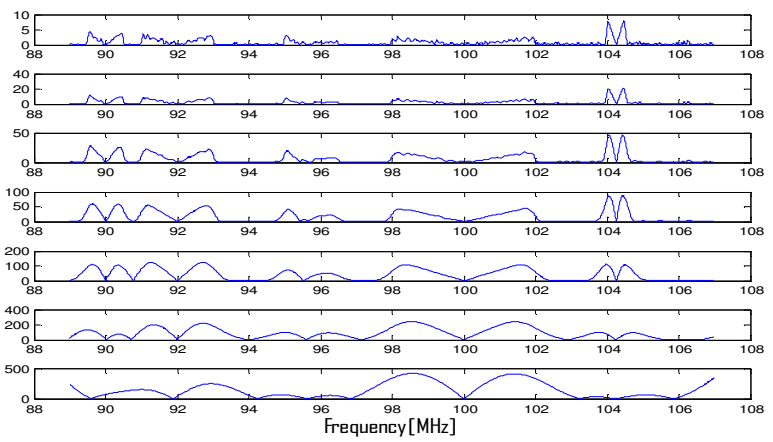

Figure 5. CWT for various scales from top to bottom( $\mathrm{S}=2,4,8,16,32,64,128)$. For this simulation a Gaussian mother-wavelet $\Phi_{\text {gaus }}$ was used.
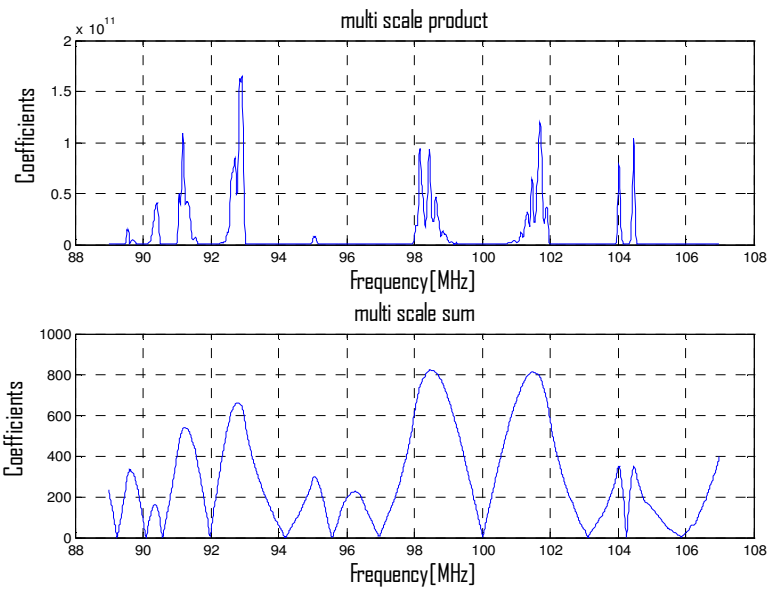

Figure 6. Multiscale product (top); Multiscale sum (bottom)

It can be observed from these plots that the Edge detection performance of wavelets with higher scales is better. This is because the slow variation appears when the singularity of signal PSD is decreased. And these edges can be characterized by wavelet modulus maxima on high scale. After analysis, the following results were obtained: 
i) For slow varying peaks, wavelet with a higher scale is advisable.

ii) For abrupt peaks, wavelet with a smaller scale is advisable.

For the proposed method, the same spectrum was used. Adaptively, the coefficients from both the transforms were taken according to the nature of the peaks. The threshold, $\delta$ was taken as 10 $\mathrm{db}$. The combined Multi scale sums for the Haar mother wavelet system, $\Phi_{\text {Haar }}$ and the Gaussian mother wavelet system, $\Phi_{\text {Gaus }}$ was used to determine the spectrum boundaries. The obtained boundary set is [89.6, 90.38, 91.2, 92.82, 95.08, 96.26, 98.44, 101.5, 104, 104.5] MHz. It can be observed that this technique yields accurate results even when the spectrum is characterized by lobes that are of different shapes and sizes. The results are shown in Figure 7.

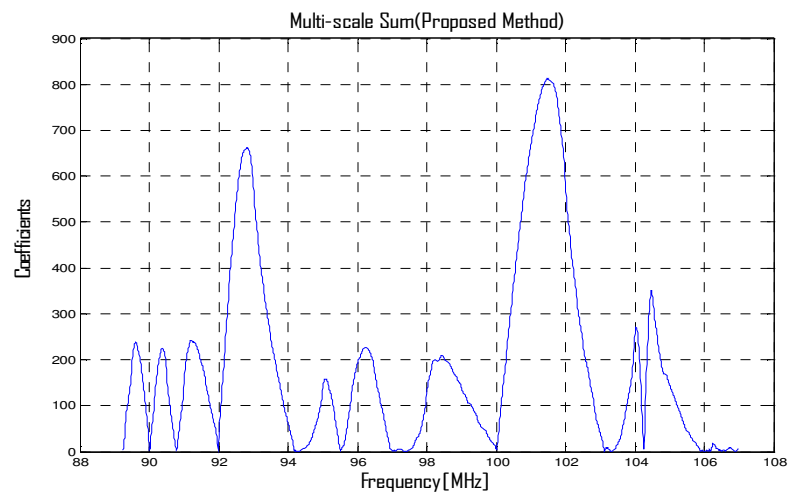

Figure 7. The plot of Multi scale sum obtained by using the proposed method.

\section{CONCLUSION}

In this paper, a detailed analysis of the Wavelet based Edge Detection technique has been presented. The scope of utilizing this property of Wavelets finds application not only in the field of Spectrum Sensing, but also in many Signal and Image processing applications, where discontinuities must be studied. Also, a number of traditional spectrum sensing methods have been investigated, and their drawbacks are pointed out. Also the incorporation of the unique features of Wavelets in Spectrum Sensing has been explained. An overview of different approaches using wavelets for precise distinction between occupied and unoccupied spectrum bands at their boundaries is presented. The reader is expected to understand the pros and cons of each technique according to the nature and dynamics of the spectrum bands and also the practical devices he/she is working with. Finally, a new algorithm is proposed for adaptive selection of the mother wavelet system according to the spatial properties of the spectrum. This is one area where much focus has not been provided. By choosing the exact type of wavelet system to match the nature of the spectra, it is possible to alleviate the computational burden of analysing finer scales. With less stringent requirements from the practical side, this efficient spectrum sensing algorithm can be used for accurate detection of discontinuities in the frequency bands. Future works include the exploration of Agent-based modelling techniques for Co-operative spectrum sensing [20] in Ad-Hoc networks, and also the cyclostationary feature based classification of dynamic spectra.

\section{ACKNOWLEDGEMENTS}

We would like to thank our Project mentor Mr. R. Gandhiraj, Asst. Professor (SR), for his invaluable assistance and support throughout the course of this undertaking. We would also like to thank the Department of ECE, Amrita Vishwa Vidyapeetham, for providing us with the facility for carrying out the simulations. 
International Journal of Computer Science \& Information Technology (IJCSIT), Vol 3, No 2, April 2011

\section{REFERENCES}

[1] Mallat,S.Zhong,S."Characterization of signals from multiscale edges" Pattern Analysis and Machine Intelligence, IEEE Transactions onVolume:14, Issue:7 Publication Year: 1992 , $\operatorname{Page}(s): 710-732$

[2] Adoum B.A, Jeoti V, "Cyclostationary Feature Based Multiresolution Spectrum Sensing Approach for DVB-T and Wireless Microphone Signals”, International Conference on Computer and Communication Engineering, pp.1 - 6, 2010

[3] K. Kim, I. A. Akbar, K. K. Bae, J. Um, C. M. Spooner and J. H. Reed, "Cyclostationary approaches to signal detection and classification in cognitive radio," in Proc. IEEE Dynamic Spectrum Access Nets, 2007, pp. 212-215.

[4] B. M. Sadler, A. Swami, "Analysis of Multiscale Products for Step Detection and Estimation”, IEEE Trans. on Inform. Theory, vol. 45, no. 3 PP. 1043-1051, April 1999.

[5] Yun-Lin Xu, Hong-Shun Zhang, Zhao-Hui Han, "The performance analysis of spectrum sensing algorithms based on wavelet edge detection" Wireless Communications, Networking and Mobile Computing, 2009. WiCom'09. $5^{\text {th }}$ International Conference on Issue Date: 24-26 Sept. 2009 On page(s): $1-4$

[6] J.G. Proakis and D. G. Manolakis, Digital Signal Processing: Principles, Algorithms, and Applications, Fourth Edition, Prentice Hall, Inc, 2007.

[7] B. Porat, A Course in Digital Signal Processing. New York: John Wiley and Sons, Inc., 1996.

[8] D. Cabric, S. M. Mishra, and R. W. Brodersen, "Implementation Issues in Spectrum Sensing for Cognitive Radios”, Proceedings of the Asilomar Conference on Signals and Systems, 2004.

[9] William A. GARDNER, "The Spectral Correlation Theory of Cyclostationary Time-series", Signal Processing 11 (1986) 13-36 13North-Holland.

[10] Bakhit Amine Adoum and Varun Jeoti, "Cyclostationary Feature Based Multiresolution Spectrum Sensing Approach for DVB-T and Wireless Microphone Signals". International Conference on Computer and Communication Engineering (ICCCE 2010), 11-13 May 2010, Kuala Lumpur, Malaysia.

[11] D. J. Thomson, "Spectrum Estimation and Harmonic Analysis", Proc. IEEE, vol. 70, no. 9, pp.1055-1096, September 1982.

[12] M. Narendar, A. P. Vinod, A.S. Madhukumar and Anoop Kumar Krishna, "An Algorithm for Spectrum Sensing in Cognitive Radio using Tree-Structured Filter Bank”, 17th International Conference on Telecommunications, 2010.

[13] Sugreev Kaur and Rajesh Mehra, “High Speed and Area Efficient 2D DWT Processor based Image Compression”, Signal \& Image Processing : An International Journal(SIPIJ) Vol.1, No.2, December 2010.

[14] K.P.Soman, K.I.Ramachandran, "Insight Into Wavelets: From Theory to Practice", $2^{\text {nd }}$ Edition, Prentice-Hall of India, 2005.

[15] De Almeida E.P.L, De Carvalho, P.H.P, Cordeiro, P.A.B, Vieira, R.D, “ Experimental Study of a Wavelet-based Spectrum Sensing Technique”, 42nd Asilomar Conference on_Signals, Systems and Computers,pp 1552-1556, 2008

[16] Lakshmanan M.K, Nikookar.H, “A Review of Wavelets for Digital Wireless Communication”, Wireless Personal Communications (2006) 37: 387-420.

[17] M.K.Lakshmanan, D.D.Ariananda and H.Nikookar, "Cognitive Radio Transmission and Spectrum Sensing using a Wavelet Packet Transceiver", IEEE $20^{\text {th }}$ International Symposium on Personal, Indoor and Mobile Radio Communication, 2009

[18] Zhi Tian, Georgios B. Giannakis," Compressed Sensing for Wideband Cognitive Radios”, IEEE International conference on Acoustics, Speech and Signal Processing, 2007.

[19] S. S. Chen, D. L. Donoho, and M. A. Saunders, "Atomic decomposition by basis pursuit", 
International Journal of Computer Science \& Information Technology (IJCSIT), Vol 3, No 2, April 2011

SIAMJ Sci. Comput., vol. 20, no. 1, pp. 33-61, 1999.

[20] Rajagopal Sreenivasan, Sasirekha GVK and Jyotsna Bapat, "Adaptive Threshold based on Group Decisions for Distributed Spectrum Sensing in Cognitive Adhoc Networks", $2^{\text {nd }}$ International

Conference on Wireless and Mobile Networks (WiMoNe 2.0), January 2011.

\section{AUTHORS PROFILE}

Karthik Divakaran is currently pursuing senior year B.Tech (2007-2011) Electronics and Communication Engineering in Amrita Vishwa Vidyapeetham, Coimbatore, Tamil Nadu. His areas of interest include Software Defined Radio and Microwave Engineering.

Naveen Manikandan is currently pursuing senior year B.Tech (2007-2011) Electronics and Communication Engineering in Amrita Vishwa Vidyapeetham, Coimbatore, Tamil Nadu. His areas of interest include Mobile communications and MIMO systems.

Shri Hari is currently pursuing senior year B.Tech (2007-2011) Electronics and Communication Engineering in Amrita Vishwa Vidyapeetham, Coimbatore, Tamil Nadu. His areas of interest include wireless communication systems and cognitive radios. 